\title{
QUANTIFICAÇÃO DE CANABINOIDES EM EXTRATOS MEDICINAIS DE Cannabis POR CROMATOGRAFIA LÍQUIDA DE ALTA EFICIÊNCIA
}

\author{
Virgínia M. Carvalho ${ }^{a, *,(i)}$, Andrey F. L. Aguiara, Leopoldo C. Baratto ${ }^{\mathrm{b}}$, Fábio L. C. Souza ${ }^{\mathrm{a}}$ e Ernesto D. Rocha ${ }^{\mathrm{a}}$ \\ aDepartamento de Análises Clínicas e Toxicológicas, Faculdade de Farmácia, Universidade Federal do Rio de Janeiro, 21941-902 \\ Rio de Janeiro - RJ, Brasil \\ bDepartamento de Produtos Naturais e Alimentos, Faculdade de Farmácia, Universidade Federal do Rio de Janeiro, 21941-902 \\ Rio de Janeiro - RJ, Brasil
}

Recebido em 07/06/2019; aceito em 25/09/2019; publicado na web em 08/01/2020

\begin{abstract}
CANNABINOIDS QUANTIFICATION IN MEDICINAL Cannabis EXTRACTS BY HIGH PERFORMANCE LIQUID CHROMATOGRAPHY. Cannabidiol (CBD) and tetrahydrocannabinol (THC) are the main active principles in the Cannabis extracts. Rich-CBD extracts are indicated to control seizures in epileptic patients, THC is indicated as antiemetic to patients in chemotherapy treatment and extracts with THC and CBD in proportions of approximately 1:1 are indicated to control spasticity in patients with multiple sclerosis. In Brazil epileptic pediatric patients are treated with imported Cannabis extracts registered as food supplement and with national not regulated Cannabis extracts. With the medical Cannabis sanitary regulation in Brazil, quality control methods will be required. A High Performance Liquid Chromatography (HPLC) method was developed and validated for five cannabinoids quantification in oily extracts and resins. The method was applied to 101 Cannabis extracts consumed by patients attended by a risk assessment program. Linearity, selectivity and precision met the validation criteria of national and international sanitary agencies. Cannabis imported oily extracts showed high CBD levels $\left(72.53 \pm 60.63 \mathrm{mg} \mathrm{mL}^{-1}\right)$ and low THC levels $\left(1.21 \pm 1.32 \mathrm{mg} \mathrm{mL}^{-1}\right)$, while national Cannabis extracts showed mainly two profiles, first composed by negative or cannabinoids traces and the second composed by THC rich extracts $\left(9.81 \pm 13.49 \mathrm{mg} \mathrm{mL}^{-1}\right)$. The HPLC method is useful to pharmaceutical production and risk assessment purposes.
\end{abstract}

Keywords: Cannabis extracts; HPLC; quality control; cannabinoids levels

\section{INTRODUÇÃO}

Extratos medicinais de espécies de Cannabis vêm sendo utilizados no tratamento de diversas condições patológicas, mas os resultados clínicos no tratamento da epilepsia refratária tiveram papel de destaque na regulamentação sanitária nacional. ${ }^{1}$ Embora Cannabis sativa L. seja a espécie principal do gênero Cannabis, diversas subespécies e inúmeras variedades de planta são cultivadas. ${ }^{2}$

Diversos compostos formados no metabolismo secundário desta planta são de interesse farmacológico, especialmente os canabinoides (terpenofenólicos). Os compostos majoritários são o ácido tetrahidrocanabinólico (THCA) e ácido canabidiólico (CBDA), que quando convertidos às suas formas neutras tetrahidrocanabinol (THC) e o canabidiol (CBD), apresentam efeitos farmacológicos paradoxais no Sistema Nervoso Central. O THC é psicoativo com propriedades euforizantes, enquanto o CBD é depressor com propriedades anticonvulsivante e ansiolítica. ${ }^{3} \mathrm{O}$ THC ainda apresenta efeito antiemético e analgésico e o CBD efeito antipsicótico e anti-inflamatório. ${ }^{4}$

As proporções de THC e CBD são empregadas na América do Norte e Europa para classificar a Cannabis como droga/maconha (drug type/marijuana) ou como fibra/cânhamo (fyber type/hemp), e assim variedades com teores de THC superiores ao de CBD são classificadas como maconha e sujeitas a maior controle devido à propriedades psicoativas do THC, enquanto variedades com teores de CBD superiores ao de THC são classificadas como cânhamo ${ }^{2} \mathrm{e}$ permitidas na produção de fibras, suplementos alimentares e cosméticos desde que o teor de THC seja $\leq 0,3 \% .^{5}$

No Brasil a importação de extratos medicinais de Cannabis foi regulamentada pela Agência Nacional de Vigilância Sanitária (ANVISA) através da RDC No 17/2015 após o Conselho Federal de

\footnotetext{
*e-mail: vmcfarm@gmail.com
}

Medicina (CFM), Resolução RE Nº 2.113/2014, autorizar a prescrição compassiva de canabidiol para tratamento de epilepsias refratárias aos tratamentos convencionais. Em 2016 a ANVISA incluiu na autorização de importação para uso médico pessoal, através da RDC 66/2016, a planta Cannabis sativa L., partes da planta e de seus compostos, incluindo o THC. Um ano depois foi registrado no Brasil o medicamento Mevatyl ${ }^{\circledR}$, indicado para controle da espasticidade na esclerose múltipla, constituído por extrato hidroalcoólico de Cannabis sativa $\mathrm{L}$. contendo $27 \mathrm{mg} \mathrm{mL}^{-1}$ de THC e $25 \mathrm{mg} \mathrm{mL}^{-1}$ de CBD, além de outros canabinoides minoritários e terpenos. Devido ao teor mais alto de THC em relação ao CBD, este medicamento é contra-indicado para controle de convulsões e para pacientes menores de 18 anos.

A matéria-prima empregada na produção dos extratos medicinais de Cannabis são as sumidades floridas de espécimes pistiladas (fêmeas) ricas em THCA e/ou CBDA que são submetidas ao aquecimento para descarboxilação dos canabinoides às suas formas neutras obtendo-se assim os princípios ativos THC e CBD. Dependendo das condições de armazenamento, da temperatura e tempo de descarboxilação, a matéria-prima pode conter o produto de degradação do THC, o canabinol $(\mathrm{CBN}) .{ }^{6}$ Esses cinco canabinoides são os marcadores empregados no controle de qualidade de produtos medicinais (Figura 1).

Atualmente os extratos de Cannabis utilizados no Brasil no tratamento de epilepsias refratárias e outros quadros patológicos são os produtos importados registrados em seu país de origem como suplementos alimentares e produtos nacionais obtidos no mercado ilícito ou elaborados artesanalmente por familiares de pacientes. ${ }^{7}$

Segundo os rótulos dos produtos importados, o veículo mais empregado é o triglicerídeo de cadeia média (TCM), enquanto na preparação artesanal, a resina de Cannabis é geralmente dissolvida em óleos de oliva, de coco ou de girassol.

A regulamentação do uso de extratos medicinais de Cannabis e a necessidade de fiscalização e monitoramento com fins de saúde 
<smiles>CCCCCc1cc2c(c(O)c1C(=O)O)[C@H]1CC(C)=CC[C@H]1C(C)O2</smiles><smiles>C=C(C)[C@H]1CCC(C)=C[C@H]1c1c(O)cc(CCCCC)c(C(=O)O)c1O</smiles>

\section{Ácido tetrahidrocanabinólico (THCA)}

\section{Ácido canabidiólico (CBDA)}

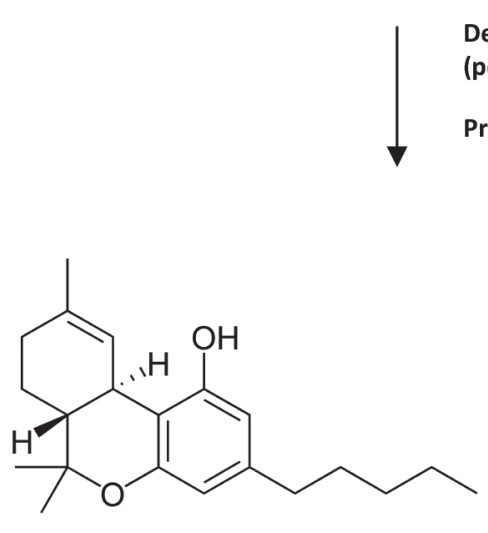

Tetrahidrocanabinol (THC)

\author{
Descarboxilação por aquecimento \\ (perda de $\mathrm{CO}_{2}$ )
}

Preparação da matéria-prima

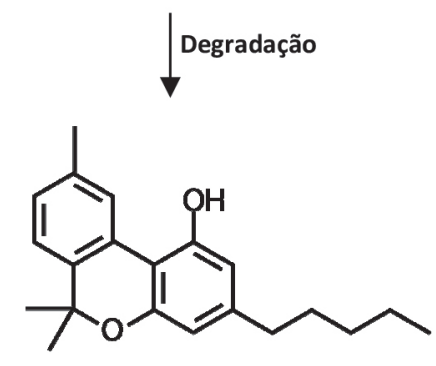<smiles>C=C(C)[C@H]1CCC(C)=C[C@H]1c1c(O)cc(CCCCC)cc1O</smiles>

Canabidiol (CBD)

\section{Canabinol (CBN)}

Figura 1. Canabinoides de interesse no controle de qualidade de extratos medicinais de Cannabis

pública justificam a padronização de métodos de controle de qualidade aplicáveis ao insumo farmacêutico ativo vegetal (IFAV) e aos extratos formulados em veículos oleosos. Assim, o objetivo desse trabalho foi desenvolver e validar um método de controle de qualidade para os canabinoides aplicável ao IFAV e aos extratos medicinais de Cannabis formulados em matrizes oleosas.

\section{PARTE EXPERIMENTAL}

\section{Material e reagentes}

Materiais de referência certificados THC, CBD, THCA, CBDA e CBN nas concentrações de $1 \mathrm{mg} \mathrm{mL}^{-1}$ (Cerilliant ${ }^{\circledR}$ ) foram empregados na otimização e validação do método. Diazepam, pureza de 99,9\% fornecido pelo Instituto Nacional de Controle de Qualidade em Saúde/ Fiocruz (Brasil), foi utilizado como padrão interno. Metanol e água ultrapura (grau HPLC) $\left(\right.$ Scharlau $\left.^{\circledR}\right), n$-hexano grau HPLC $\left(\right.$ Tedia $\left.^{\circledR}\right)$. Solução tampão formiato de amônio $(50 \mathrm{mM}, \mathrm{pH} 5,19)$ foi preparada com formiato de amônio (Loba Chemie ${ }^{\circledR}$ ) e ajustado o pH com ácido fórmico $\left(\right.$ Tedia $\left.^{\circledR}\right)$.

\section{Amostragem e preparação das amostras}

Extratos medicinais de Cannabis $(\mathrm{n}=101)$ foram doados por pacientes atendidos pelo projeto de extensão universitária Farmacannabis no período de fevereiro de 2018 a dezembro de 2018 (estudo aprovado pelo Comitê de Ética em Pesquisa do Hospital Universitário Clementino Fraga Filho, No CAAE 2021817.0.00005257).

Alíquotas de $100 \mathrm{mg}$ para amostras em veículo oleoso e $20 \mathrm{mg}$ para amostras de resinas (IFAVs) foram transferidas para tubos de polipropileno e adicionados $10 \mathrm{~mL}$ do solvente metanol: $n$-hexano $(9: 1 \mathrm{v} / \mathrm{v})$ contendo padrão interno na concentraçao de $0,005 \mathrm{mg} \mathrm{mL}^{-1}$. As amostras foram homogeneizadas a $2000 \mathrm{rpm}$ por 1 min e submetidas ao banho de ultrassom (US) por $30 \mathrm{~min}$. As amostras em veículo oleoso foram refrigeradas a $-20^{\circ} \mathrm{C}$ por 30 min e centrifugadas a 4000 rpm por $20 \mathrm{~min}$. As amostras de resinas diluídas e homogeneizadas por US e os sobrenandantes das amostras centrifugadas foram filtrados em membrana PTFE de 0,22 $\mu$ m e transferidos para vials de HPLC e adotada a diluição apropriada de acordo com o perfil cromatográfico, por exemplo, para os extratos em torno de $50 \mathrm{mg} \mathrm{mL}^{-1}$, o fator de diluição final foi de 200 vezes. 


\section{Condições cromatográficas}

Equipamento de cromatografia líquida de alta eficiência (CLAE) acoplado ao detector de arranjo de diodos $\left(\right.$ Thermo $\left.^{\circledR}\right)$, dotado de bomba quartenária Rheos 5600, automostrador Accela e detector PDA Accela de $20 \mathrm{~Hz}$. Os dados foram processados em software ChromQuest 5.0.

O método foi desenvolvido em coluna cromatográfica, marca ACE, dimensões $250 \times 4,6 \mathrm{~mm}$ e partículas de $5 \mu \mathrm{m}$ em temperatura constante de $30^{\circ} \mathrm{C}$. Os analitos foram eluídos no modo gradiente empregando como solvente A tampão formiato de amônio, $50 \mathrm{mM}$, pH 5,19, e solvente B metanol nas seguintes condições: 0-10 min de 32 a $15 \%$ de A; 10 a 20 min de 15 a $8 \%$ de A; 20 a 22 min de 8 a $5 \%$ de $\mathrm{A}, 22$ a 25 min 5\% de $\mathrm{A} ; 25$ a 30 min retornou-se à condição inicial de $32 \%$ de A. O fluxo foi de $1,0 \mathrm{~mL} / \mathrm{min}$ e injeção de $10 \mu \mathrm{L}$. A detecção foi realizada em comprimento de onda de $240 \mathrm{~nm}$.

Os canabinoides foram identificados por seus espectros em comparação com os padrões de referência e pelos tempos de retenção.

\section{Validação do método}

Os parâmetros avaliados foram linearidade, seletividade, precisão, limite de detecção (LD), limite de quantificação (LQ) e exatidão de acordo com as recomendações exaradas pela ANVISA, RDC $N^{\circ} 166 / 2017^{8}$, pela Instrução Normativa No $4 / 2014$ que define os critérios para registro de controle de qualidade de fitoterápicos ${ }^{9} \mathrm{e}$ pelo International Conference on Harmonization (ICH). ${ }^{10} \mathrm{~A}$ curva de calibração foi ensaiada com 7 pontos nas concentrações de 0,001 a $0,030 \mathrm{mg} \mathrm{mL}^{-1}$ para os cinco canabinoides de interesse a partir de soluções-padrão. O LD e LQ foram determinados de acordo com o $\mathrm{ICH}^{10}$ pela análise de seis amostras branco de óleo de oliva misturado com óleo de girassol e utilizada a relação entre o desvio padrão e a resposta (área) e o slope (S) da curva de calibração. Foi empregada como critério $3,3 \sigma / \mathrm{S}$ para o LD e $10 \sigma / \mathrm{S}$ para o LQ.

A repetibilidade e precisão intermediária foram avaliadas somente para os marcadores farmacológicos THC e CBD. Os ensaios foram realizados empregando soluções padrão secundárias preparadas a partir de resina rica em CBD importada e resina rica em THC nacional diluídas em metanol para obtenção do padrão secundário de THC na concentração de $200,00 \mathrm{mg} \mathrm{mL}^{-1}$ e padrão secundário de CBD na concentração de $150,00 \mathrm{mg} \mathrm{mL}^{-1}$. Quantidades apropriadas do padrão secundário foram evaporadas em fluxo de $\mathrm{N}_{2}$ e ressuspendidas no branco de óleo de oliva misturado com óleo de girassol para preparação das amostras controle de qualidade 1 (CQ1) nas concentrações 3,50 mg mL-1 para THC e 2,50 mg mL-1 para CBD e das amostras CQ2 nas concentrações $52,00 \mathrm{mg} \mathrm{mL}^{-1} \mathrm{e} 45,00 \mathrm{mg} \mathrm{mL}^{-1}$ para THC e CBD. Assim, a repetibilidade foi ensaiada pela análise de seis replicatas das amostras $C_{1}$ e $C_{2}$ e a precisão intermediária pela análise de seis replicatas de $\mathrm{CQ}_{1}$ e $\mathrm{CQ}_{2}$ em dois dias alternados.

A exatidão do método foi mensurada como porcentagem de recuperação para os canabinoides CBD, THC e CBN nas concentrações de 1,00 e 2,00 $\mathrm{mg} \mathrm{mL}^{-1}$ na matriz TCM por ter sido este o veículo mais referido nos rótulos de produtos importados. Quantidades adequadas de materiais de referência certificados foram combinadas, o solvente evaporado e realizada a diluição dos canabinoides no branco de matriz.

Óleos comerciais foram empregados nos ensaios de seletividade, tais como óleo de coco orgânico extra-virgem, marca Copra lote 91005616; óleo de soja orgânico, marca Korin, lote TG0950 08:53:069; óleo de girassol, marca Pazze, lote 41215/6; óleo de oliva orgânico extra-virgem, marca O-live, lote 00002; e triglicerídeo de cadeia média (TCM), marca Lótus, lote 180807.

\section{RESULTADOS E DISCUSSÃO}

\section{Separação cromatográfica e validação}

A separação de cinco canabinoides (CBDA, CBD, THCA, THC e $\mathrm{CBN}$ ) e do padrão interno (diazepam) ocorreu em 21 min e apresentou boa resolução nas matrizes ensaiadas (Figura 2). Métodos por cromatografia gasosa (CG) e CLAE são descritos na literatura para determinação de canabinoides em diferentes matrizes, além de outros métodos ainda pouco difundidos como a cromatografia em fluido supercrítico. ${ }^{11}$

Os métodos por $\mathrm{CG}$, apesar de rápidos e robustos, requerem derivatização dos canabinoides ácidos devido às altas temperaturas requeridas na operação. ${ }^{12,13-15}$ Assim, quando a análise de canabinoides se destina ao controle de qualidade de produtos farmacêuticos e suplementos alimentares, os métodos por CLAE representam a melhor

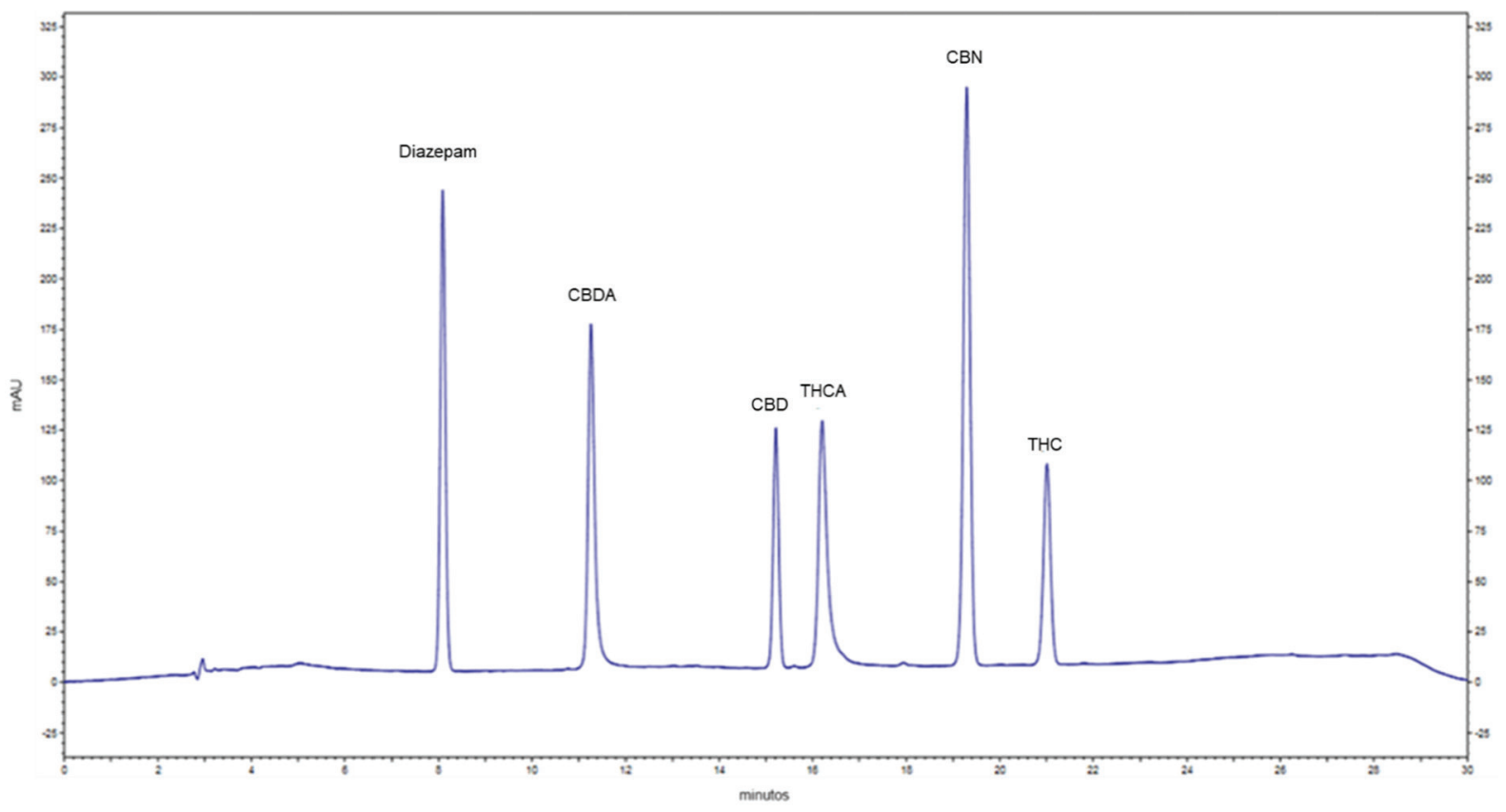

Figura 2. Perfil cromatográfico dos analitos CBDA, CBD, THCA, CBN, THC nas concentrações de 0,020 $\mathrm{mg} \mathrm{mL}^{-1}$ e do padrão interno diazepam (0,005 $\mathrm{mg}$ mL $\left.L^{-1}\right)$ 
opção ${ }^{16,17,18}$ porque não requerem aquecimento, sendo aplicável aos canabinoides ácidos e neutros simultaneamente, permitindo verificar se a descarboxilação requerida na preparação do insumo farmacêutico ativo vegetal foi adequada.

Dentre os métodos por CLAE publicados, ${ }^{12,14,17,19,20,21}$ o descrito por De Backer et al. (2009) ${ }^{12}$ foi citado no artigo de revisão de Citti et al. $(2018)^{17}$ como o mais referido na literatura e selecionado para otimização e validação neste trabalho. Os fatores que nortearam essa escolha foram (1) boa resolução para os analitos de interesse THC, CBD, CBDA, THCA e CBN; (2) emprego do metanol como solvente orgânico na fase móvel que tem menor custo em relação à acetonitrila e (3) uso do detector de arranjo de diodos que também apresenta menor custo de aquisição e manutenção em relação à espectrometria de massas. No trabalho original ${ }^{12}$ foi utilizado prazepam como padrão interno, no entanto, como essa substância não é produzida no Brasil, optou-se pelo diazepam, referido por Citti et al. (2016) ${ }^{16}$ e cujo material de referência é produzido e fornecido pelo Instituto Nacional de Controle de Qualidade em Saúde da Fiocruz.

Apesar do método selecionado ${ }^{12}$ ter sido aplicado a amostras vegetais empregando coluna $\mathrm{C} 18$ com menor tamanho de partícula $(2 \mu \mathrm{m})$ para melhor resolução dos picos, a coluna empregada neste trabalho com tamanho de partícula de $5 \mu \mathrm{m}$ e o gradiente modificado mostraram-se adequados para a separação dos cinco canabinoides no IFAV e nos extratos formulados em matrizes oleosas conservando-se a sequência de eluição dos picos.

A padronização de métodos para quantificação de canabinoides apresenta alto custo no Brasil porque os padrões de referência precisam ser importados e estão sob estrito controle internacional. Esses materiais de referência certificados são fornecidos em ampolas de $1 \mathrm{~mL}$, concentração de $1 \mathrm{mg} \mathrm{mL}^{-1}$ com valores em torno de U\$ 350 para os canabinoides THC, CBD e CBN e U\$ 700 para os canabinoides ácidos THCA e CBDA, que são instáveis e necessitam de transporte refrigerado com armazenamento a $-20^{\circ} \mathrm{C}$. Apesar dos teores de canabinoides nos extratos medicinais serem, em teoria, na ordem de algumas dezenas de miligramas, parte da validação do método teve que ser realizada na ordem de microgramas, visto a solução estoque do material de referência certificado apresentar teor de 1,0 $\mathrm{mg} \mathrm{mL}^{-1}$. A maior dificuldade de implantar no Brasil o método de controle de qualidade para canabinoides consiste no alto custo dos materiais de referência certificados, burocracia de importação e demora na entrega.

Outra dificuldade técnica para desenvolvimento e validação de método, quando o foco é a avaliação de segurança dos tratamentos ou fiscalização de produtos, é a grande variabilidade de veículos empregados na preparação dos extratos medicinais. Enquanto os rótulos dos extratos importados declararam TCM como veículo, os extratos artesanais foram preparados com óleo de oliva, óleo de girassol, óleo de coco, óleo de soja e alguns pacientes relataram misturas de óleos. Ainda alguns extratos fornecidos no mercado nacional não regulado continham em seus rótulos a descrição de glicerina como veículo.

No ensaio de seletividade, as matrizes que apresentaram picos interferentes de maior intensidade com os mesmos tempos de retenção do CBDA, CBD e THC (Tabela 1) foram óleo de oliva e óleo girassol (Figuras 3 e 4).

Sendo o presente método proposto para controle de qualidade e avaliação de segurança dos extratos medicinais de Cannabis cujos teores esperados dos marcadores farmacológicos (THC e CBD) são na ordem de $\mathrm{mg} \mathrm{mL}^{-1}$, optou-se pela mistura das matrizes óleo de oliva e óleo de girassol para obtenção do branco utilizado nos ensaios de LD, LQ e precisão.

Tabela 1. Interferentes nos tempos de retenção dos analitos de interesse

\begin{tabular}{lccc}
\hline \multirow{2}{*}{ Matrizes } & \multicolumn{3}{c}{ Concentração de interferentes $\left(\mathrm{mg} \mathrm{mL}^{-1}\right)$} \\
\cline { 2 - 4 } & CBD & CBDA & THC \\
\hline Óleo de coco & 0,07 & 0,08 & $\mathrm{ND}$ \\
Óleo de soja & 0,05 & 0,08 & $\mathrm{ND}$ \\
Óleo de girassol & $\mathrm{ND}$ & 0,06 & 0,05 \\
Óleo de oliva & 0,07 & 0,09 & $\mathrm{ND}$ \\
TCM & 0,07 & 0,08 & $\mathrm{ND}$ \\
\hline
\end{tabular}

TCM: triglicerídeo de cadeia média; ND: não detectado.

Os resultados dos parâmetros linearidade, LD, LQ, precisão e exatidão (Tabelas 2, 3 e 4) atenderam a finalidade de controle de qualidade dos produtos medicinais. A precisão foi avaliada somente para THC e CBD porque esses são os marcadores farmacológicos

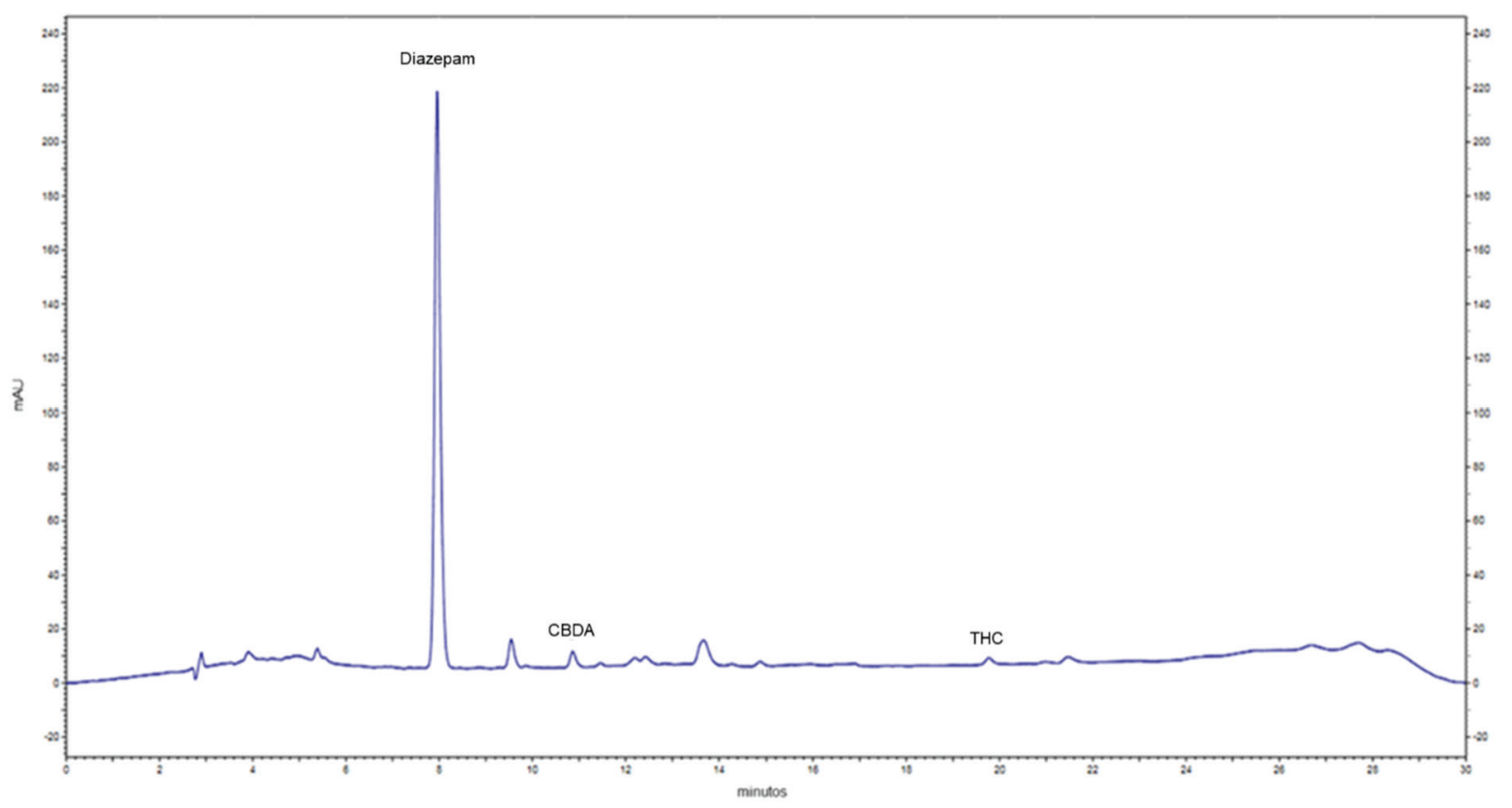

Figura 3. Perfil cromatográfico do óleo de girassol 


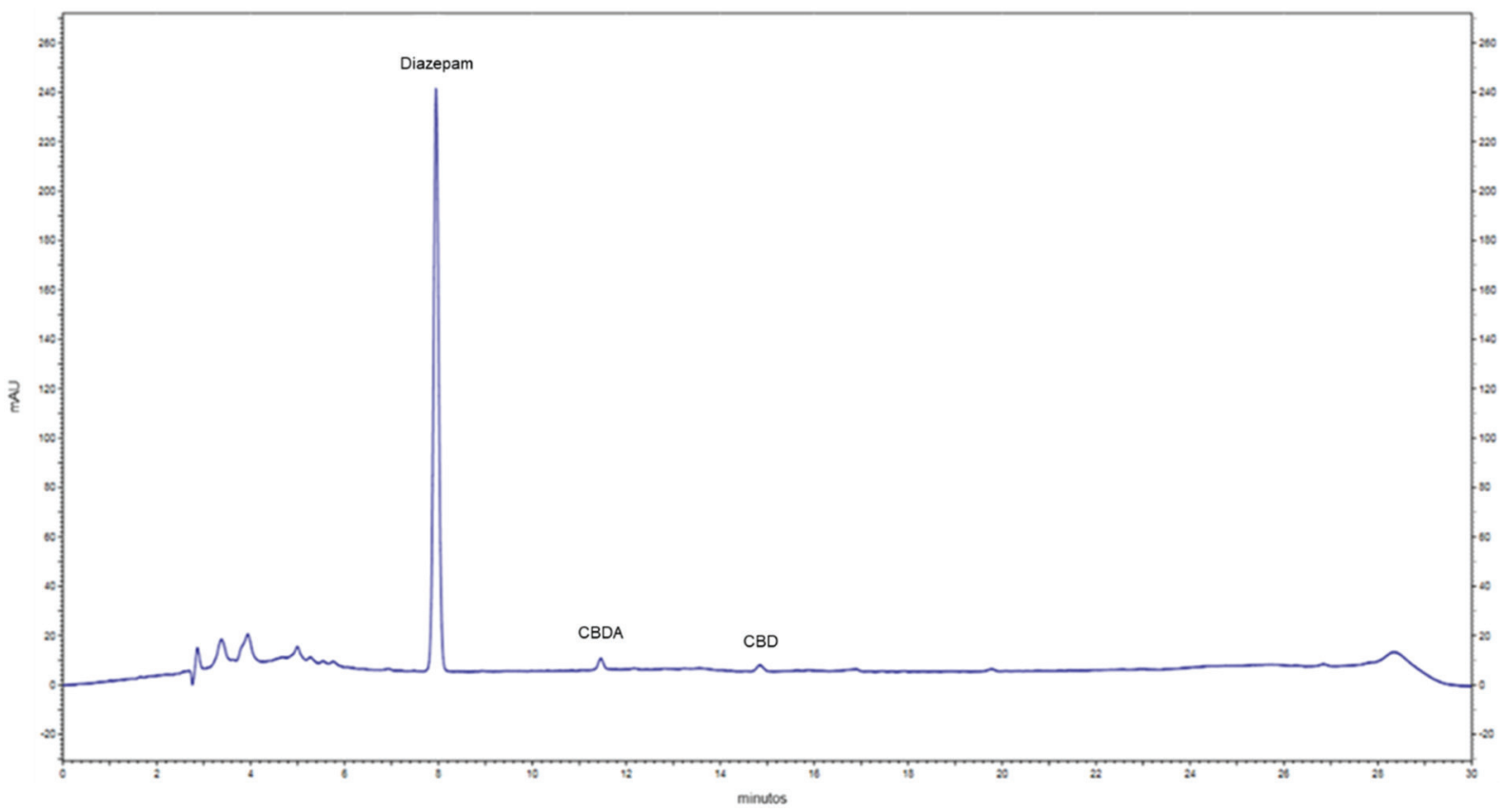

Figura 4. Perfil cromatográfico do óleo de oliva

Tabela 2. Resultados dos ensaios de linearidade, LD e LQ

\begin{tabular}{ccccc}
\hline Analito & Equação da reta & $\mathrm{r}^{2}$ & LD & LQ \\
\hline THC & $\mathrm{y}=5,5402 \mathrm{x}+0,0996$ & 0,999365 & 0,22 & 0,66 \\
CBD & $\mathrm{y}=6,9874 \mathrm{x}+0,1077$ & 0,999651 & 0,20 & 0,59 \\
THCA & $\mathrm{y}=3,7752 \mathrm{x}+0,1589$ & 0,998592 & 0,15 & 0,44 \\
CBDA & $\mathrm{y}=3,7436 \mathrm{x}+0,1129$ & 0,999396 & 0,11 & 0,35 \\
CBN & $\mathrm{y}=2,1120 \mathrm{x}+0,0802$ & 0,999548 & 0,09 & 0,26 \\
\hline
\end{tabular}

Tabela 3. Resultados dos ensaios precisão

\begin{tabular}{lccccc}
\hline & THC & & \multicolumn{2}{c}{ CBD } \\
\cline { 2 - 3 } \cline { 5 - 6 } & $3,50 \mathrm{mg} \mathrm{mL}^{-1}$ & $52,00 \mathrm{mg} \mathrm{mL}^{-1}$ & & $2,50 \mathrm{mg} \mathrm{mL}^{-1}$ & $45,00 \mathrm{mg} \mathrm{mL}^{-1}$ \\
\hline Repetibilidade (CV\%) & 6,47 & 2,97 & 3,25 & 6,21 \\
Precisão intermediária (CV\%) & 5,89 & 7,24 & 5,43 & 6,42 \\
\hline
\end{tabular}

Tabela 4. Recuperação do método

\begin{tabular}{cccc}
\hline Concentração $\left(\mathrm{mg} \mathrm{mL}^{-1}\right)$ & CBD & THC & CBN \\
\hline 1,00 & $89,51 \%$ & $85,05 \%$ & $97,09 \%$ \\
2,00 & $117,45 \%$ & $99,49 \%$ & $90,34 \%$ \\
\hline
\end{tabular}

dos extratos medicinais, a presença dos canabinoides ácidos THCA e CBDA indica que a técnica de descarboxilação na preparação da matéria-prima foi inadequada e a presença de CBN indica a degradação do THC. Assim considera-se que o método foi completamente validado apenas para os marcadores farmacológicos THC e CBD.

Os valores do coeficiente de variação obtidos nos ensaios de precisão atenderam ao critério da Instrução Normativa $N^{\circ} 4 / 2014$ para produtos fitoterápicos $(\mathrm{CV} \leq 15 \%){ }^{9}$

O método foi aplicado aos extratos importados em veículos oleosos $(n=26)$ e resinas $(n=7)$ e aos extratos nacionais em veículos oleosos $(n=62)$ e resinas $(n=6)$. Os extratos oleosos importados apresentaram como principal veículo o TCM, enquanto os extratos oleosos nacionais utilizaram óleo de oliva, óleo de coco, glicerina, óleo de soja e óleo de girassol. Os extratos importados eram todos suplementos alimentares, a maioria importada dos Estados Unidos da América (EUA), enquanto os extratos nacionais, segundo relato dos responsáveis dos pacientes, foram obtidos em diferentes regiões do Brasil tais como Sul (Santa Catarina), Sudeste (São Paulo, Rio de Janeiro e Minas Gerais) e Nordeste (Paraíba e Piauí), sendo que $15 \%$ das amostras nacionais foram produzidas em casa pelos responsáveis de pacientes. As amostras apresentaram alta variabilidade nas características organolépticas.

O perfil cromatográfico dos extratos analisados norteou o planejamento das diluições a serem empregadas na fase final de preparação da amostra. Todos os extratos importados apresentaram perfil predominante do canabinoide CBD (Figura 5) com teores cerca de 100 vezes acima do que foi determinado nos extratos nacionais cujo canabinoide majoritário foi o CBD. Os extratos nacionais apresentaram diferentes perfis, podendo ser classificados primordialmente como extratos com perfil predominante do canabinoide THC (Figura 6) com origens da região Sul e Sudeste e traços de canabinoides formulados em glicerina cujas origens relatadas foram o Estado da Paraíba, Piauí e Minas Gerais.

A alta variabilidade dos teores de canabinoides, principalmente nos extratos de origem nacional onde não se tem teores indicativos 
nos rótulos, dificulta o estabelecimento de um fator de diluição único. Assim para as quantificações foi necessária uma análise inicial para avaliação do perfil cromatográfico e posterior diluição com solvente de extração contendo padrão interno para que as concentrações de canabinoides ficassem dentro da curva de calibração. A falta de padronização dos extratos nacionais e de alguns produtos importados com teores muito acima ou abaixo dos valores rotulados requereu duas corridas cromatográficas para cada amostra.

A Tabela 5 apresenta os teores de canabinoides nos extratos importados e nacionais estratificados de acordo com os canabinoides majoritários. Somente 10 dos 68 extratos nacionais apresentaram perfil predominante de CBD e mesmo assim os teores foram muito abaixo dos determinados nos extratos importados. Os teores de THC superiores ao de CBD nos extratos nacionais mostram que eles são produzidos majoritariamente com variedades de plantas de Cannabis classificadas internacionalmente como maconha (marijuana).

Apesar da regulamentação americana adotar o padrão de uso ao invés da diferenciação hemp ou marijuana para determinar se a Cannabis é medicinal, ${ }^{5}$ os perfis do produtos nacionais apresentados na Tabela 5 não são comparáveis com os extratos importados e teriam uso medicinal restrito aos quadros patológicos que requerem THC, o que não é o caso das epilepsias refratárias. Além dos extratos oleosos nacionais não apresentarem teores de CBD equivalentes com os determinados nos importados, ainda apresentaram teores de CBDA e THCA maiores (Figura 7) indicando que a preparação da matéria-prima não foi apropriada, uma vez que a descarboxilação é requerida para conversão dos compostos ácidos em neutros e obtenção dos efeitos terapêuticos.

Os maiores teores de THC verificados nas amostras nacionais podem ser explicados pelo mercado da Cannabis ser direcionado ao

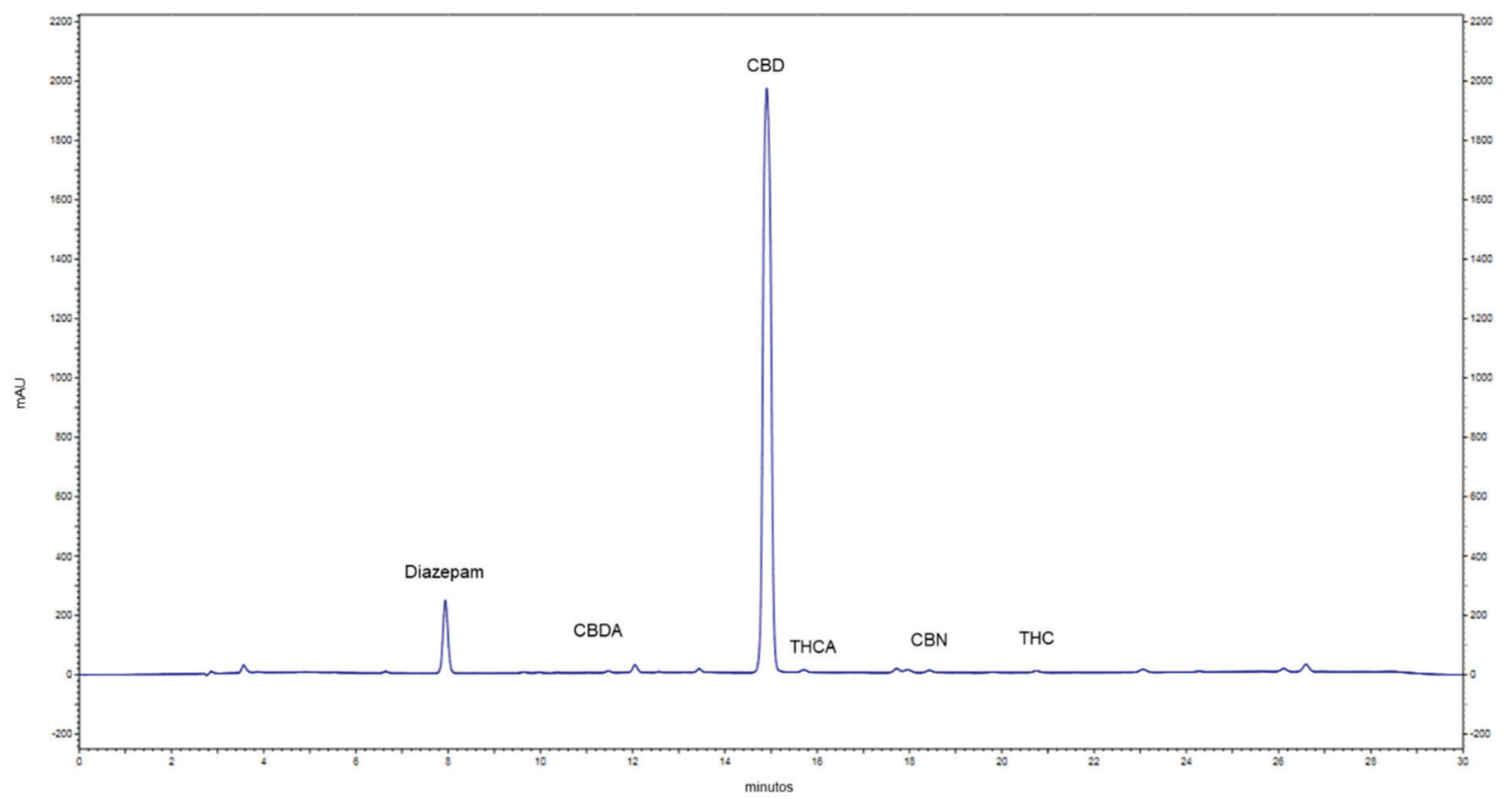

Figura 5. Perfil cromatográfico de extrato importado formulado em TCM rotulado como CBD puro

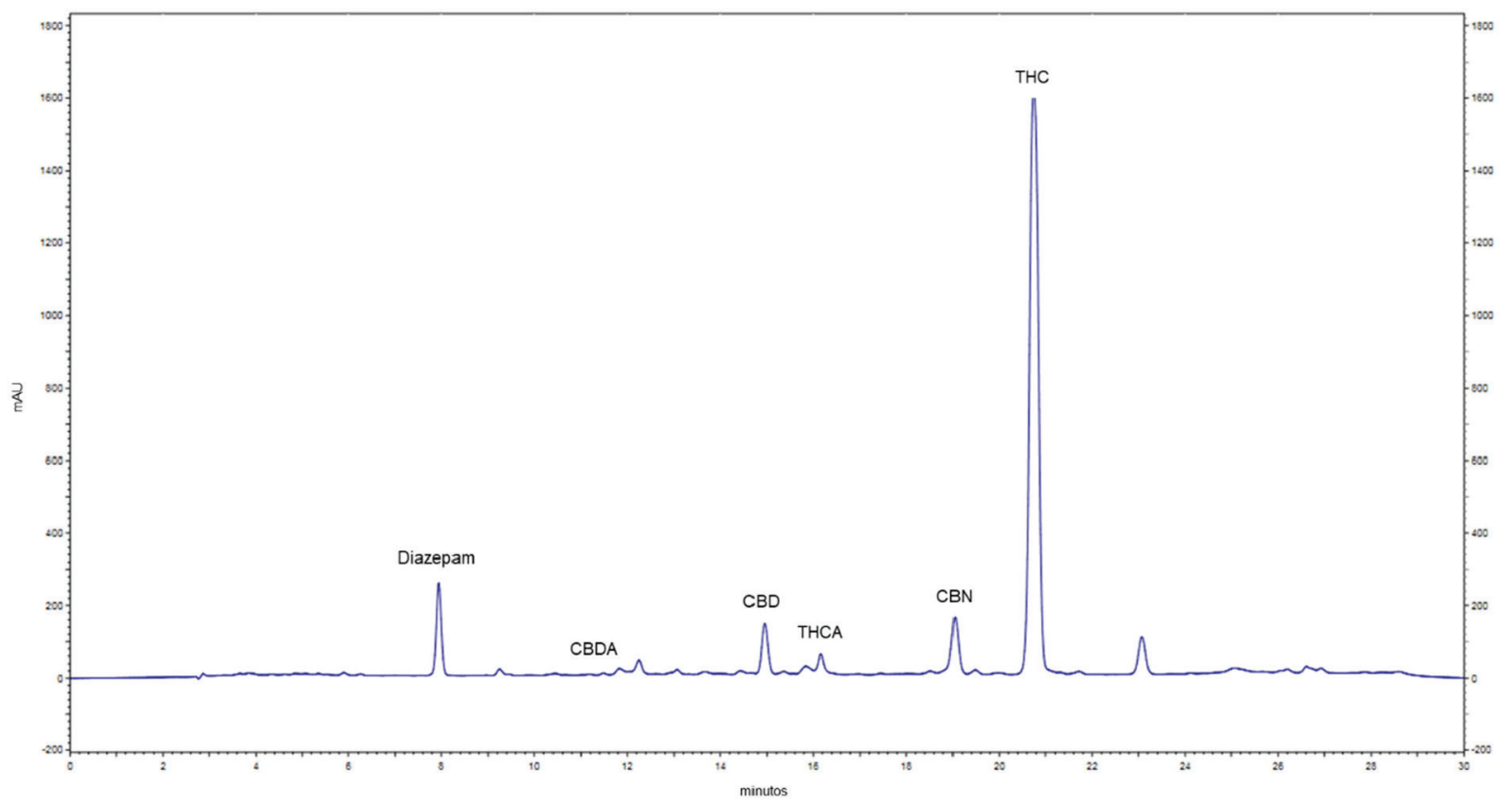

Figura 6. Perfil cromatográfico de extrato nacional formulado em óleo de oliva rico em THC 
Tabela 5. Distribuição dos canabinoides nos extratos medicinais segundo o perfil

\begin{tabular}{|c|c|c|c|c|c|}
\hline \multirow{3}{*}{ Perfil } & \multicolumn{5}{|c|}{ NACIONAIS } \\
\hline & \multicolumn{5}{|c|}{$\mathrm{mg} \mathrm{mL}^{-1}$} \\
\hline & $\mathrm{CBD}$ & THC & CBDA & THCA & $\mathrm{CBN}$ \\
\hline \multicolumn{6}{|l|}{ THC $(\mathbf{n}=30)$} \\
\hline Média \pm DP & $0,64 \pm 0,92$ & $9,81 \pm 13,49$ & $0,13 \pm 0,21$ & $1,24 \pm 1,25$ & $0,44 \pm 1,12$ \\
\hline Faixa & $0,00-2,30$ & $0,73-44,85$ & $0,00-1,02$ & $0,00-4,77$ & $0,00-1,03$ \\
\hline \multicolumn{6}{|l|}{ CBD $(n=10)$} \\
\hline Média \pm DP & $3,06 \pm 1,90$ & $0,18 \pm 0,12$ & $1,98 \pm 1,82$ & $<\mathrm{LD}$ & $<\mathrm{LD}$ \\
\hline Faixa & $0,95-10,45$ & $0,00-0,49$ & $0,00-4,44$ & $0,00-0,26$ & $0,00-0,10$ \\
\hline \multicolumn{6}{|l|}{ Traços $(n=22)$} \\
\hline Média \pm DP & $<\mathrm{LD}$ & $0,19 \pm 0,23$ & $0,14 \pm 0,15$ & $0,16 \pm 0,26$ & $<\mathrm{LD}$ \\
\hline Faixa & $0,00-0,25$ & $0,00-0,90$ & $0,00-0,46$ & $0,00-1,10$ & $0,00-0,20$ \\
\hline Resinas THC $(n=6)$ & \multicolumn{5}{|c|}{$\mathrm{mg} \mathrm{g}^{-1}$} \\
\hline Média \pm DP & $14,88 \pm 2,34$ & $293,42 \pm 57,20$ & $0,64 \pm 0,93$ & $4,87 \pm 4,00$ & $11,92 \pm 9,53$ \\
\hline \multirow[t]{4}{*}{ Faixa } & $0,33-16,99$ & $28,30-348,48$ & $0,00-2,39$ & $0,14-11,49$ & $4,20-29,56$ \\
\hline & \multicolumn{5}{|c|}{ IMPORTADOS } \\
\hline & \multicolumn{5}{|c|}{$\mathrm{mg} \mathrm{mL}^{-1}$} \\
\hline & CBD & THC & CBDA & THCA & $\mathrm{CBN}$ \\
\hline \multicolumn{6}{|l|}{ CBD $(n=26)$} \\
\hline Média \pm DP & $72,53 \pm 60,63$ & $1,21 \pm 1,32$ & $0,22 \pm 0,50$ & $0,26 \pm 0,46$ & $0,16 \pm 0,21$ \\
\hline Faixa & $13,17-284,90$ & $0,00-4,33$ & $0,00-1,78$ & $0,00-1,84$ & $0,00-0,75$ \\
\hline Resinas CBD $(n=7)$ & $\mathrm{mg} \mathrm{g}^{-1}$ & & & & \\
\hline Média \pm DP & $151,40 \pm 31,45$ & $2,91 \pm 2,46$ & $1,13 \pm 1,65$ & $1,27 \pm 1,95$ & $0,65 \pm 0,52$ \\
\hline Faixa & $104,99-189,26$ & $0,86-8,15$ & $0,00-4,49$ & $0,00-5,22$ & $0,00-1,35$ \\
\hline
\end{tabular}

DP: desvio padrão; LD: limite de detecção.

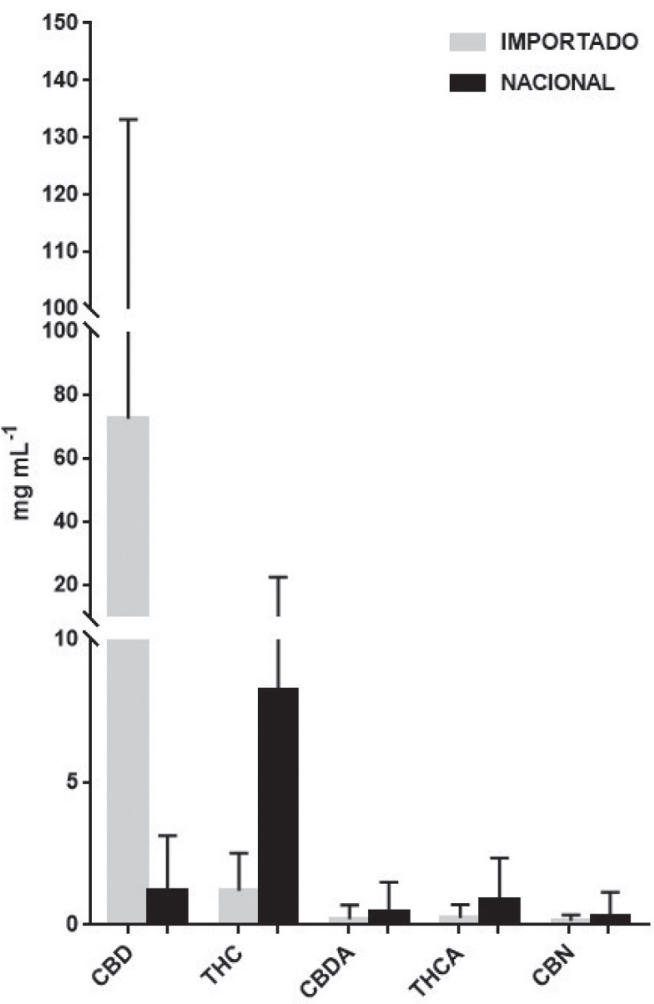

Figura 7. Comparação de médias e desvios padrão dos teores de canabinoides nos extratos oleosos consumo recreativo. Esse fenômeno pode estar ocorrendo também em outros países. Em uma avaliação de 61 extratos de Cannabis consumidos por crianças portadoras de epilepsia em uma comunidade na Austrália foi verificado que os perfis de canabinoides naqueles produtos foram semelhantes aos achados no presente estudo, prevalecendo extratos com perfil THC, teores muitos baixos de CBD ou traços de canabinoides. ${ }^{22}$

Em 2018 foi registrado o primeiro medicamento à base de CBD pela agência sanitária dos EUA (Food and Drug Administration) sob o nome comercial Epidiolex®. Este medicamento, produzido pela GW Pharma na apresentação de $100 \mathrm{mg} \mathrm{mL}^{-1}$ de CBD, é indicado para o tratamento de convulsões em pacientes a partir de 2 anos portadores das síndromes de Lennox-Gastaut e de Dravet com doses que vão de 5 a $10 \mathrm{mg} / \mathrm{kg} / \mathrm{dia}$. Se por um lado a ação do CBD no controle de convulsões associadas a determinadas síndromes está comprovada, por outro lado, pacientes que substituem protocolos terapêuticos convencionais por extratos com traços de canabinoides ou teores muito baixos de CBD podem ter seu quadro patológico agravado pela falta de medicação adequada.

Extratos ricos em THC, embora tenha indicação como tratamento paliativo no controle de dor, náuseas e vômitos em pacientes sob quimioterapia, podem agravar crises convulsivas e causar surtos psicóticos e isto foi relato por algumas mães de crianças portadoras de epilepsia atendidas no projeto Farmacannabis.

Ao contrário dos EUA e Europa que regulamentaram o cânhamo para a produção de fibras, a regulação brasileira nunca fez distinção do perfil químico entre cânhamo e maconha; por exemplo, a Portaria $\mathrm{N}^{\circ} 344 / 98$ que complementa a Lei $n^{\circ} 11.343 / 2006$, a chamada Lei de 
Drogas, aloca na lista E (plantas proibidas) a Cannabis sp. englobando todas as espécies, subespécies e variedades do gênero Cannabis. Os resultados obtidos nas amostras autênticas mostram a necessidade do investimento nacional no cultivo de variedades Cannabis de interesse médico, especialmente as de cânhamo, e no desenvolvimento de produtos, pois a falta de padronização farmacêutica dos extratos medicinais representa risco à saúde, especialmente para crianças que devido ao quadro patológico têm dificuldade de comunicar os sintomas, ficando a avaliação médica e do cuidador restrita aos sinais clínicos

\section{CONCLUSÃO}

O método mostrou-se adequado para análise de extratos formulados em diferentes veículos oleosos e em resinas e poderá ser adotado no controle de qualidade do IFAV e extratos medicinais de Cannabis.

A composição e teores de canabinoides dos produtos importados diferem dos produtos nacionais, enquanto todos os importados apresentaram perfil de extratos de cânhamo, a maioria dos produtos nacionais apresentou perfil de extratos de maconha.

$\mathrm{O}$ perfil dos extratos nacionais direcionam a necessidade de investimento nacional no cultivo de variedade de Cannabis de interesse médico, especialmente de cânhamo, e na produção farmacêutica.

\section{AGRADECIMENTOS}

Esse trabalho contou com suporte financeiro do Instituto Serrapilheira (Processo No Serra-1709-18891) e do Conselho Nacional de Desenvolvimento Científico e Tecnológico (Processo $\left.\mathrm{N}^{\circ} 426406 / 2018-4\right)$

\section{REFERÊNCIAS E NOTAS}

1. Carvalho, V. M.; Brito, M. S; Gandra, M.; Forum Sociológico 2017, 30, 57.

2. United Nations Office on Drugs and Crimes. United Nations: New York, 2009.

3. Honório, K. M.; Arroio, A.; Silva, A. B. F.; Quim. Nova 2006, $29,318$.

4. Hill, K. P. JAMA - J. Am. Med. Assoc. 2015, 313, 2474.
5. Mead, A. Epilepsy Behav.; 2017, 70, 288.

6. Lewis, M. M.; Yang, Y.; Wasilewski, E.; Clarke, H. A.; Kotra, L. P.; ACS Omega 2017, 2, 6091.

7. Carvalho, V. M.; Braz. J. Anal. Chem. 2017, 4, 44.

8. BRASIL. RDC $N^{\circ} 166$, de 24 de julho de 2017. Dispõe sobre a validação de métodos analíticos e dá outras providências. Diário $O f$. [da] República Fed. do Bras. 2017, 22.

9. ANVISA. Guia de orientação para registro de Medicamento Fitoterápico e registro e notificação de Produto Tradicional Fitoterápico. Agência Nac. Vigilância Sanitária. Instrução Norm. $n^{\circ}$ 4, 18 junho 2014. 1-123.

10. Dep. Health Hum. Serv. (U.S); Validation Of Analytical Procedures: Methodology.1997.

11. Wang, M.; Wang, Y. H.; Avula, B.; Radwan, M. M.; Wanas, A. S.; Mehmedic, Z.; Antwerp, J.; ElSohly, M. A.; Khan, I. A.; J. Forensic Sci. 2017, 62, 602 .

12. De Backer, B.; Debrus B.; Lebrun, P.; Theunis, L.; Dubois, N.; Decock, L.; Verstraete, A.; Hubert, P.; Charlier, C.; J. Chromatogr. B: Biomed. Sci. Appl. 2009, 877, 4115.

13. Patel, B.; Wene, D.; Fan, Z. T.; J. Pharm. Biomed. Anal. 2017, 146.

14. Burnier, C.; Esseiva, P.; Roussel, C.; Talanta 2019, 192, 135.

15. Casiraghi, A.; Roda, G.; Casagni, E.; Cristina, C.; Musazzi, U. M.; Franzé, S.; Rocco, P.; Giuliani, C.; Fico, G.; Minghetti, P.; Gambaro, V.; Planta Med. 2018, 84, 242.

16. Citti, C.; Ciccarella, G.; Braghiroli, D.; Parenti, C.; Vandelli, M. A.; Cannaza, G.; J. Pharm. Biomed. Anal. 2016, 128, 201.

17. Citti, C.; Battisti, U. M.; Braghiroli, D.; Ciccarella, G.; Schmid, M.; Vandelli, M. A.; Cannazza, G.; Phytochem. Anal. 2018, 29, 144.

18. Bonn-Miller, M. O.; Loflin, M. J. E.; Thomas, B. F.; Marcu, J. P.; Hyke, T.; Vandrey, R.; J. Am. Med. Assoc. 2017, 318, 2017.

19. Ambach, L.; Penitschka, F.; Broillet, A.; König, S.; Weinmann, W.; Bernhard, W. Forensic Sci. Int. 2017, 243, 107.

20. Swift, W.; Wong, A.; Li, K. M.; Arnold, J. C.; McGregor, I. S. Analysis of Cannabis Seizures in NSW, Australia: Cannabis Potency and Cannabinoid profile. PLoS One 2013, 8, 1

21. Gul, W.; Gul, S. W.; Chandra, S.; Lata, H.; Ibrahim, E. A.; Elsohly, M. A.; Planta Med. 2018, 55, 267.

22. Suraev, A.; Lintzeris, N.; Stuart, J.; Kevin, R. C.; Blackburn, R.; Richards, E.; Arnold, J. C.; Ireland, C.; Todd, L.; Allsop, D. J.; McGregor, I. S.; Sci. Rep. 2018, 8, 1. 